\title{
Evaluation of dry textile electrodes for long-term electrocardiographic monitoring
}

\author{
Milad Alizadeh-Meghrazi 1,2,8* , Binbin Ying ${ }^{3,8}$, Alessandra Schlums ${ }^{4,8}$, Emily Lam²,8, Ladan Eskandarian ${ }^{5,8}$, \\ Farhana Abbas ${ }^{6,8}$, Gurjant Sidhu ${ }^{7,8}$, Amin Mahnam ${ }^{8}$, Bastien Moineau ${ }^{8}$ and Milos R. Popovic ${ }^{1,2}$
}

${ }^{*}$ Correspondence:

milad.alizadeh.m@utoronto.

ca

${ }^{1}$ KITE Research Institute, Toronto Rehabilitation

Institute - University Health Network (UHN), Toronto, ON,

Canada

Full list of author information is available at the end of the article

\begin{abstract}
Background: Continuous long-term electrocardiography monitoring has been increasingly recognized for early diagnosis and management of different types of cardiovascular diseases. To find an alternative to $\mathrm{Ag} / \mathrm{AgCl}$ gel electrodes that are improper for this application scenario, many efforts have been undertaken to develop novel flexible dry textile electrodes integrated into the everyday garments. With significant progresses made to address the potential issues (e.g., low signal-to-noise ratio, high skin-electrode impedance, motion artifact, and low durability), the lack of standard evaluation procedure hinders the further development of dry electrodes (mainly the design and optimization).
\end{abstract}

Results: A standard testing procedure and framework for skin-electrode impedance measurement is demonstrated for the development of novel dry textile electrodes. Different representative electrode materials have been screen-printed on textile substrates. To verify the performance of dry textile electrodes, impedance measurements are conducted on an agar skin model using a universal setup with consistent frequency and pressure. In addition, they are demonstrated for ECG signals acquisition, in comparison to those obtained using conventional gel electrodes.

Conclusions: Dry textile electrodes demonstrated similar impedance when in raised or flat structures. The tested pressure variations had an insignificant impact on electrode impedance. Looking at the effect of impedance on ECG signals, a noticeable effect on ECG signal performance metrics was not observed. Therefore, it is suggested that impedance alone is possibly not the primary indicator of signal quality. As well, the developed methods can also serve as useful guidelines for future textile dry-electrode design and testing for practical ECG monitoring applications.

Keywords: Dry textile electrodes, Electrophysiological monitoring,

Electrocardiography (ECG), Textile computing, Long-term biosignal monitoring, Remote healthcare, Printed electronics, Carbon-contained yarn, Silver-plated yarn

\section{Background}

Biopotentials or electrophysiological signals are widely utilized in health monitoring and diagnostics. For example, through electrocardiography (ECG), cardiac activity is recorded and information regarding potential cardiovascular diseases (CVD) (e.g., heart

(C) The Author(s) 2021. This article is licensed under a Creative Commons Attribution 4.0 International License, which permits use, sharing, adaptation, distribution and reproduction in any medium or format, as long as you give appropriate credit to the original author(s) and the source, provide a link to the Creative Commons licence, and indicate if changes were made. The images or other third party material in this article are included in the article's Creative Commons licence, unless indicated otherwise in a credit line to the material. If material is not included in the article's Creative Commons licence and your intended use is not permitted by statutory regulation or exceeds the permitted use, you will need to obtain permission directly from the copyright holder. To view a copy of this licence, visit http://creativecommons.org/ licenses/by/4.0/. The Creative Commons Public Domain Dedication waiver (http://creativecommons.org/publicdomain/zero/1.0/) applies to the data made available in this article, unless otherwise stated in a credit line to the data. 
rhythm abnormalities including atrial fibrillation) can be obtained [1]. To record ECG signals, biopotential electrodes are used, which are classified as invasive, that puncture the skin, or non-invasive, that do not puncture skin. With invasive electrodes, the extracellular and intracellular fluids serve as an electrolytic medium [2] for improved signal acquisition. Due to the nature of these electrodes, requiring the puncturing of the skin, they are not ideal for long-term continuous monitoring. As well, they present a further limitation with implementation, requiring the supervision of a trained professional. With non-invasive electrodes, an electrolytic gel can be used, or they can be used without electrolytic gel, considered as dry contact electrodes. Currently in standard clinical settings, $\mathrm{Ag} / \mathrm{AgCl}$ gel electrodes are mainly used for recording ECG signals. These types of electrodes use a conductive gel that acts as an electrolyte between the electrode and the epidermis layer of skin, and reduces the contact impedance between them [3]. As well they have an adhesive material around the perimeter of the electrode, that helps with adherence to skin, reducing movement of the electrode, improving signal quality during motion. However, issues such as skin irritation [4] over time, and decay of signal quality due to gel dehydration $[5,6]$, hinder them from continuous and long-term wearable monitoring usage. Alternatives to the hydrogel adhered electrodes can be realized through the manual application of electrolytic hydrogel, or electrolytic pastes. These solutions leave a feasibility gap with their application and durability, limiting their consideration for continuous monitoring. As the importance of continuous long-term ECG monitoring is increasingly recognized for early diagnosis and management of different types of CVD, significant effort has been devoted to the development of skin-friendly dry electrodes for wearable biopotential measurements [7-12].

Flexible textile dry electrodes have been recently the focus of many researches, with the aim to convert biopotential monitoring to a nonintrusive process achieved with the comfort of the everyday garments that we are already using $[9,10]$. ECG electrodes are mainly fabricated using different conductive materials, such as metals [13-16], intrinsically conductive polymers (ICPs) [8, 17], and carbon-based materials [18-23]. These conductive materials and yarns can be integrated into fabrics through conventional manufacturing methods (e.g., knitting, weaving, embroidery) [24-26], or can be applied onto textiles through various techniques (e.g., stenciling, screen printing, and sputtering) [27]. Many efforts have been undertaken to optimize those novel dry electrodes, addressing issues such as weak signal pick-up, low signal-to-noise ratio, high skin-electrode impedance, motion artifact, as well as biocompatibility, and durability [10]. Among them, impedance reduction is one of the most intensively studied research directions $[7,28$, 29]. In dry contact electrodes, high skin-electrode contact impedance is observed. This is primarily due to the lack of electrolytic gel or skin preparations at the electrode application site, such as removing hair or abrading with sand paper and cleaning with alcohol wipes (lightly removing the stratum corneum). The electrical circuit model for the skinelectrode interface can be represented by resistive and capacitive components (Fig. 1) [2]. Both electrolytic gel and skin preparation are meant to reduce the skin-electrode contact impedance, which have demonstrated to improve signal acquisition quality, although it is unknown if low electrode impedance alone can guarantee high-quality biopotential recording. For example, organic conductive poly(3,4-ethylenedioxythiophene)poly(styrenesulfonate) and Ionic Liquid PEDOT:PSS + IL gel were directly printed on 


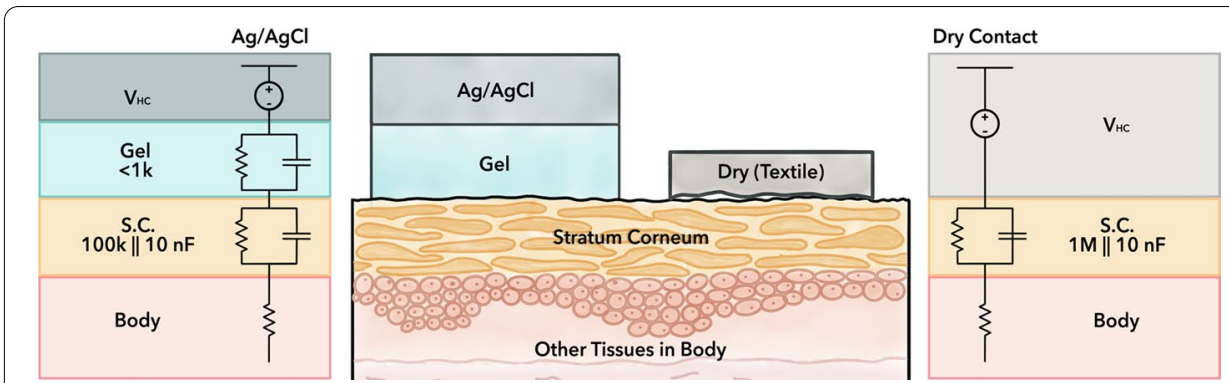

Fig. 1 Electrode-skin interface with a gel electrode vs. $\mathbf{b}$ dry contact electrode. Demonstrating differences in stratum corneum impedance electrical model when exposed to the moisture of electrolyte gel [31]

knitted textiles, showing a low contact impedance, and was utilized for long-term ECG recording [8]. With a variety of dry textile electrodes proposed by different research labs to date (Additional file 1: Table S1), it is very difficult to understand which methods and materials lead to high-performance textile electrodes that can potentially be used in practical "out of the lab" situations. This is mainly because previous studies have usually utilized different testing platforms and parameters (e.g., different textile substrates, fabrication methods, conductive coating materials, applied pressures, frequencies, skin characteristics, and ambient conditions) to evaluate and compare electrodes. Thus, we need a universal/standard evaluation protocol for electrode design and selection efficiently in the practical application scenarios [30].

In this paper, we aim to compare the electrical performance of various existing dry textile electrodes using a universal setup with consistent frequency, pressure, and skin model. Different representative electrode materials [e.g., carbon, IL, polydimethylsiloxane (PDMS)/(PEDOT:PSS), PDMS/PEDOT:PSS/IL, PDMS/PEDOT:PSS/carbon nanotube $(\mathrm{CNT})]$ reported previously have been deposited onto a flat/raised silver or carbon textile substrates by screen printing. Screen-printing was utilized for electrode fabrication because it is a simple and versatile technique well-suited and commonly used for mass production in the textile industry. To verify the performance of dry textile electrodes, impedance measurements were conducted on an agar skin model, while ECG signals were also acquired, comparing the signal fidelity to those obtained using conventional gel electrodes. These results can serve as useful guidelines for future textile-based dry-electrode design and practical ECG monitoring applications.

\section{Results}

\section{Dry textile electrode structures and their coated conductive materials}

In this study, several different materials were used to create electrodes using screen printing on flat and raised, silver and carbon knitted electrodes. Then the impedance of these electrodes to a skin model was measured, and ECG was recorded by these electrodes, to compare their performances to gel electrodes as well as bare silver and carbon electrodes. Silver-plated nylon and carbon-contained nylon conductive yarns (Myant Inc., Canada) were knitted into four different structures, namely, flat textile electrodes made of silver yarn (FS Sample code), raised 3D textile electrodes made of silver yarn (RS Sample code), flat textile electrodes made of carbon yarn (FC Sample code) and 
raised 3D textile electrodes made of carbon yarn ( $R C$ Sample code) using a flatbed knitting machine (Stoll CMS ADF 32W E7.2, Reutlingen, Germany).

Each textile electrode has a circular structure with a diameter of $2 \mathrm{~cm}$, matching the active area of the hydrogel electrodes. Figure 2 shows a schematic of knitted textile swatches and the physical appearance of the flat/raised textile electrodes. A knitted silver-plated nylon trace/cable was used as a connection line between the knitted flat/ raised electrode area and an electrode snap located on the back of the sample (Fig. 2a, b). Figure $2 \mathrm{c}-\mathrm{f}$ shows images of the flat and raised textile electrodes made of conductive silver and carbon yarns, respectively.

Thereafter, different conductive pastes (20 types) were screen-printed on these 4 types of textile electrodes (namely FS, RS, FC, and RC, shown in Fig. 2c-f). Details of conductive pastes and formulations are described in "Methods" section.

The performance of dry textile electrodes for biopotential monitoring is determined by a variety of factors (Table 1 and Additional file 1: Table S2), e.g., textile electrode substrates and structures, conductive coating materials for screen printing, applied pressures, and frequencies. In this section, we first explored the effect of electrode structures and coating conductive materials on the fabrication of dry textile electrodes. The dry textile electrodes were manufactured as flat or raised 3D structures (Fig. 2c-f). The flat structure allows for uniform pressure distribution, in line with the base fabric construction. Conversely, the raised 3D structures are protrusions from the base fabric construction leading to higher contact pressure, which could result in improved signal acquisition properties [11]. The microscopic images of FS (Fig. 2c) and FC (Fig. 2e) are shown in Fig. 3a, b. The microscopic images of RS (Fig. 2d) and RC (Fig. 2f) are shown in Fig. 4a, b, respectively. We can find a similar microscopic morphology between flat and raised 3D textile electrodes made of silver yarn (Fig. 3a, b),

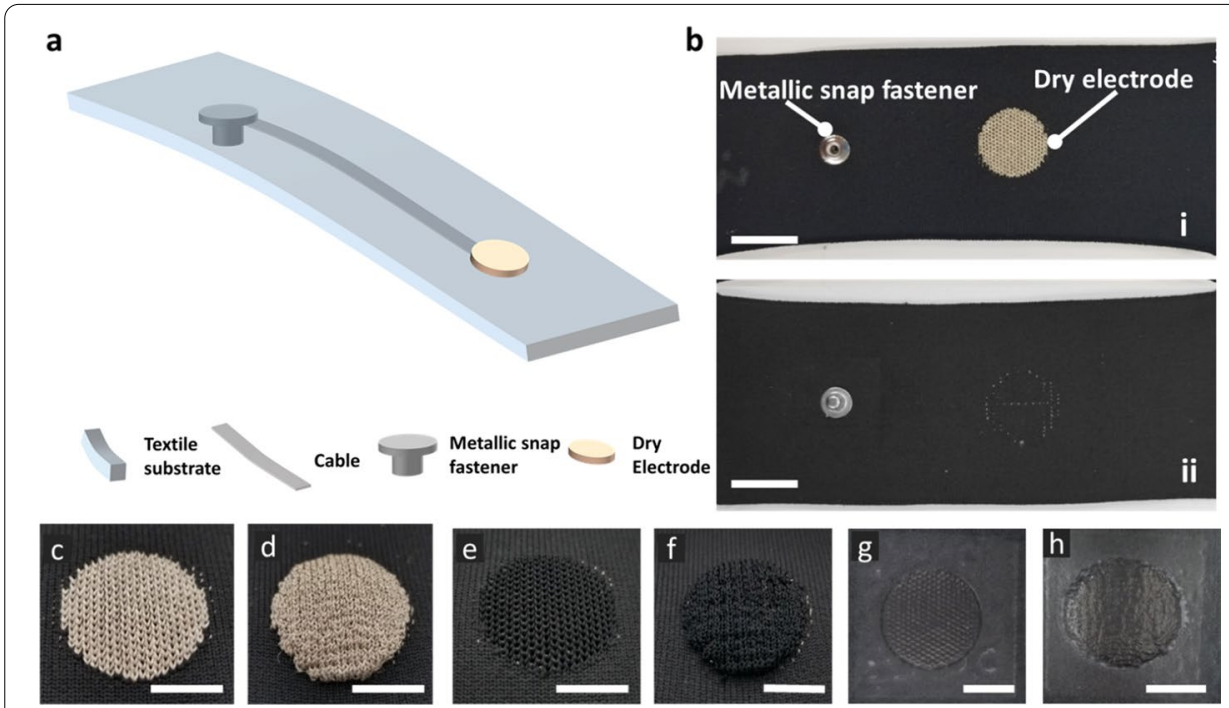

Fig. 2 Dry textile electrodes. a Schematic of a dry textile electrode. $\mathbf{b}$-i The front and $\mathbf{b}$-ii back of a sample dry textile electrode. Scale bar is $2 \mathrm{~cm}$. c Flat textile electrode made of silver yarn (FS). $\mathbf{d}$ Raised 3D textile electrode made of silver yarn (RS). e Flat textile electrode made of carbon yarn (FC). $\mathbf{f}$ Raised 3D textile electrode made of carbon yarn (RC). $\mathbf{g}$ Flat textile electrode with screen-printed coating. $\mathbf{h}$ Raised 3D textile electrode with screen-printed coating. Scale bar is $1 \mathrm{~cm}$ 
Table 1 Coating formulations successfully screen-printed onto dry textile electrodes

\begin{tabular}{|c|c|c|c|}
\hline Coating material composition & Layers & Heat curing & Pass/fail \\
\hline Carbon & 3 & $140^{\circ} \mathrm{C}, 2 \mathrm{~min}$ & Pass on $\mathrm{FS}, \mathrm{RS}, \mathrm{FC}$, and $\mathrm{RC}^{\mathrm{a}}$ \\
\hline Carbon $+2.5 \% \mathrm{IL}^{\mathrm{b}}$ & 3 & $140^{\circ} \mathrm{C}, 2 \mathrm{~min}$ & Pass on FS, RS, FC, and RC \\
\hline Carbon $+5 \% \mathrm{IL}$ & 3 & $140^{\circ} \mathrm{C}, 2 \mathrm{~min}$ & Pass on FS, RS, FC, and RC \\
\hline Carbon $+7.5 \% \mathrm{IL}$ & 3 & $140^{\circ} \mathrm{C}, 2 \mathrm{~min}$ & $\begin{array}{l}\text { Pass on } F S \text { and } F C \\
\text { Fail on } R S \text { and } R C\end{array}$ \\
\hline PEDOT:PSS + 12.5\% PDMS & 2 & $150^{\circ} \mathrm{C}, 10 \mathrm{~min}$ & $\begin{array}{l}\text { Pass on FS } \\
\text { Fail on } R S, F C \text {, and } R C\end{array}$ \\
\hline PEDOT:PSS + 6.25\% PDMS & 2 & $150^{\circ} \mathrm{C}, 10 \mathrm{~min}$ & $\begin{array}{l}\text { Pass on FS } \\
\text { Fail on } R S, F C \text {, and } R C\end{array}$ \\
\hline (PEDOT:PSS + 6.25\% PDMS) + 2.5\% IL & 2 & $150^{\circ} \mathrm{C}, 10 \mathrm{~min}$ & $\begin{array}{l}\text { Pass on FS } \\
\text { Fail on } R S, F C \text {, and } R C\end{array}$ \\
\hline (PEDOT:PSS + 6.25\% PDMS) + 5\% IL & 2 & $150^{\circ} \mathrm{C}, 10 \mathrm{~min}$ & $\begin{array}{l}\text { Pass on } F S \\
\text { Fail on } R S, F C \text {, and } R C\end{array}$ \\
\hline PEDOT:PSS + $12.5 \%\left(\right.$ PDMS + 1\% CNT) ${ }^{c}$ & 2 & $150^{\circ} \mathrm{C}, 10 \mathrm{~min}$ & $\begin{array}{l}\text { Pass on } F S \\
\text { Fail on } R S, F C \text {, and } R C\end{array}$ \\
\hline PEDOT:PSS + 6.25\% (PDMS + 1\% CNT) & 2 & $150^{\circ} \mathrm{C}, 10 \mathrm{~min}$ & $\begin{array}{l}\text { Pass on FS } \\
\text { Fail on RS, FC, and RC }\end{array}$ \\
\hline
\end{tabular}

${ }^{a} F S$ flat textile electrodes made of silver yarn, $R S$ raised $3 D$ textile electrodes made of silver yarn, $F C$ flat textile electrodes made of carbon yarn, $R C$ raised $3 D$ textile electrodes made of carbon yarn

${ }^{\mathrm{b}}$ Carbon-based coatings were mixed with $2.5 \% \mathrm{IL}$

C PEDOT:PSS-based coatings were mixed with 6.25\% PDMS and 1\% CNT
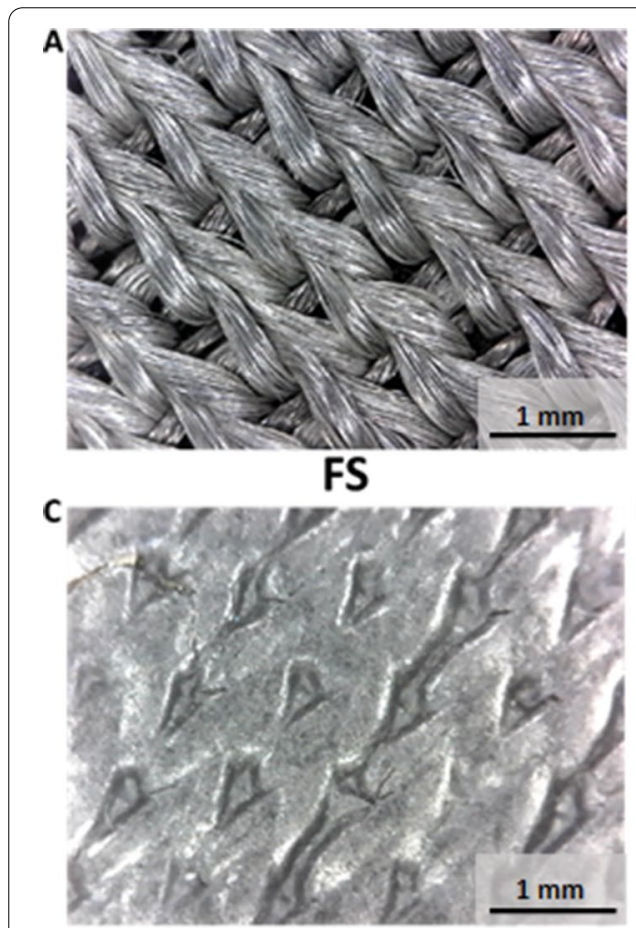

FS with carbon+2.5\%IL

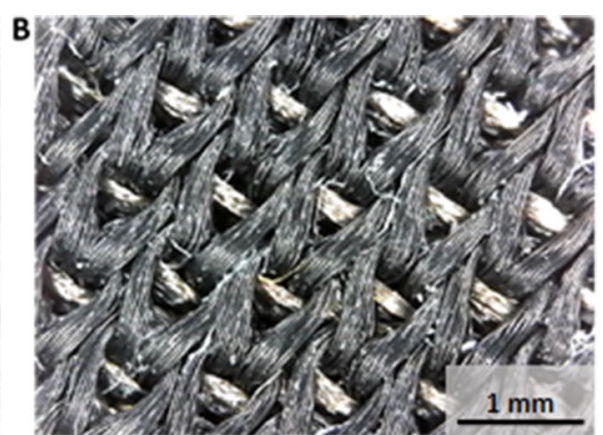

FC

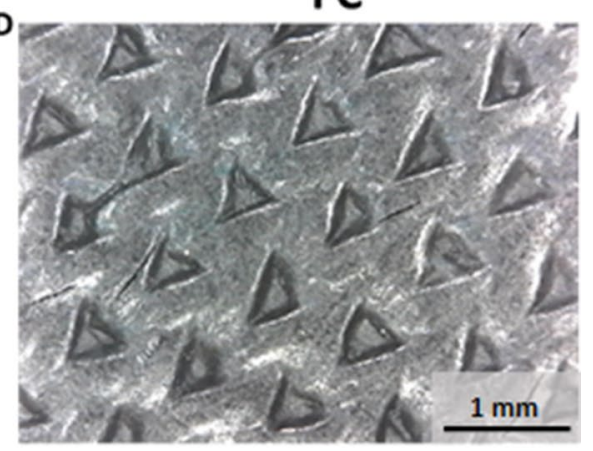

FC with carbon+2.5\%IL

Fig. 3 Microscopic images of dry textile electrodes. a FS structure. b FC structure. c FS structure with carbon $+2.5 \% \mathrm{lL}$ coating. $\mathbf{d}$ FC structure with carbon $+2.5 \% \mathrm{lL}$ coating 


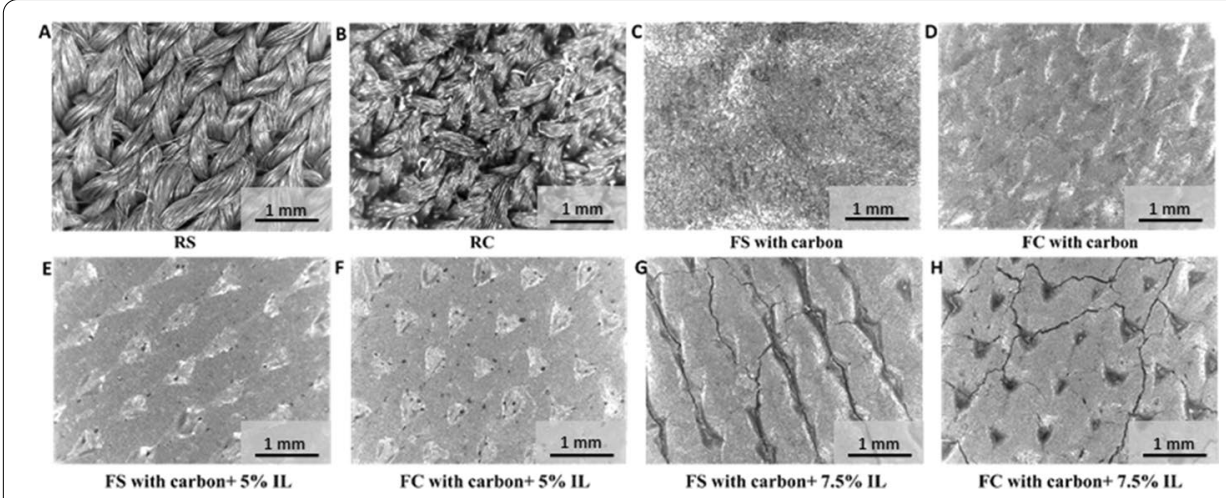

Fig. 4 Microscopic images of textile electrodes. a RS structure. b RC structure. $\mathbf{c}$ FS structure with carbon coating. $\mathbf{d}$ FC structure with carbon coating. e FS structure with carbon + 5\% lL coating. $\mathbf{f} F C$ structure with carbon + 5\%lL coating. $\mathbf{g}$ FS structure with carbon + 7.5\% l coating. $\mathbf{h}$ FC structure with carbon + 7.5\% lL coating

but a significant morphology difference between flat and raised 3D textile electrodes made of carbon yarn (Fig. 4a, b). Thereafter, we screen-printed conductive materials (listed in Table 1 and Additional file 1: Table S2) on these four types of textile substrates. Carbon-contained conductive coating materials (with/without IL) showed good printability on flat structures without obvious cracks formation (Figs. 3c, d, $4 \mathrm{c}-\mathrm{f})$. However, carbon-contained conductive coating materials with high concentrations of IL (e.g., 7.5\%) showed cracks after screen printing on the flat textile substrates (Fig. $4 \mathrm{~g}, \mathrm{~h}$ ) and failed on the raised 3D textile substrates, which was excluded from implementation. The PEDOT:PSS + PDMS-contained conductive coating materials with/without CNTs or with a suitable concentration of IL (e.g., $\leq 5 \%$ ) only showed printability without mechanical cracking on the flat textile substrates made of silver yarn. In contrast, the other combinations of PEDOT:PSS + PDMS-contained conductive coating materials listed in Additional file 1: Table S2, all failed when printed on the four textile substrates. For example, pristine PEDOT:PSS and PEDOT:PSS + IL could not be printed due to the observed mechanical cracks. This poor printability is due to the lack of flexibility and stretchability of PEDOT:PSS [32], which can be strengthened by applying biocompatible additives such as PDMS. In this scenario, we observed that PEDOT:PSS + PDMS-contained conductive coating materials without or with proper concentration of IL (e.g., $\leq 5 \%$ ) could be printed on the flat textile substrates made of silver yarn. With further increase of IL concentration (e.g., 7.5\%), this coating was observed not printable on the flat textile substrates made of silver yarn.

\section{Impedance of dry textile electrodes}

Electrode impedance has been considered as one of the main performance metrics in evaluating electrodes for recording high-quality biosignals [7, 28, 29]. We evaluated the impedance performance of dry textile electrodes with and without conductive materials (listed in Table 1) screen-printed on them. An agar skin model was utilized to mimic human skin for consistency in the testing of the electrodes and the avoidance of intra- and inter-subject skin impedance variations [33]. 


\section{Effect of frequency and coating materials}

The agar-electrode impedance of different dry textile electrodes at a widely accepted frequency range (e.g., $1 \mathrm{~Hz} \sim 10 \mathrm{kHz}[7,9]$ ) is shown in the bode plots (Fig. 5a, b and Additional file 1: Figure S3). All the tests were conducted under $20 \mathrm{mmHg}(\sim 2.66 \mathrm{kPa})$ (a typical pressure for wearable ECG measurement [34, 35]). The contact impedance decreased with the increase of frequency, which follows the typical trend of the electrical impedance measured on skin [36].

We then compared the agar-electrode impedance of dry textile electrodes with different conductive coating materials. Flat textile electrodes made of silver yarn were selected for comparison purposes since all conductive materials listed in Table 1 could be successfully printed on this type of textile substrate without cracks formation. As shown in Fig. 5a, the flat textile electrodes made of silver yarn with carbon-contained coating presented a much higher impedance than those with PEDOT:PSS-contained coating, showing a similar trend as previously reported [7]. In addition, the impedance decreasing rate highly depended on the coating conductive materials. Dry textile electrodes with carbon-contained coatings showed a much larger impedance change than that with PEDOT:PSS-contained coatings (Fig. 5a). Dry textile electrodes with PEDOT:PSS-contained coatings showed a comparable impedance level with that of standard gel electrodes (Fig. 5a, d). This could be explained by two facts: (a) carbon has a much higher sheet resistance than PEDOT:PSS [7]; (b) PEDOT:PSS can provide
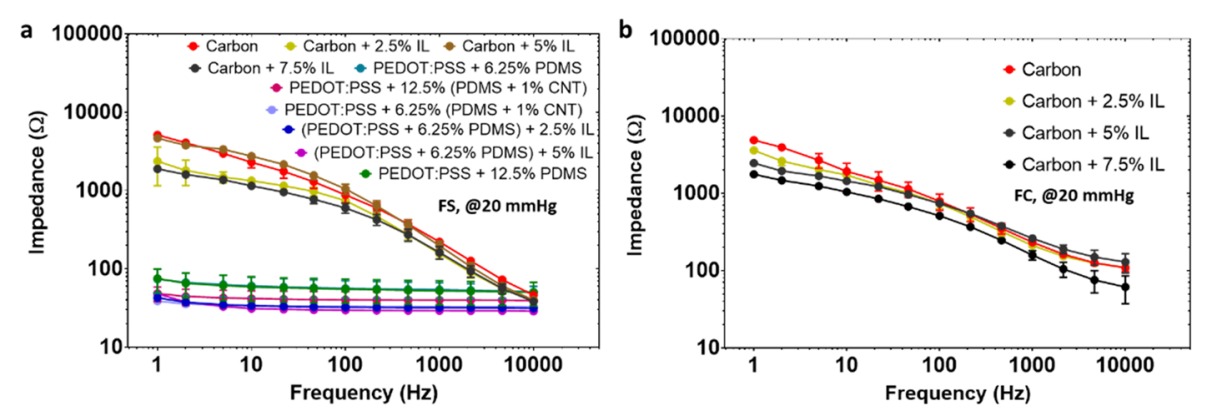

C

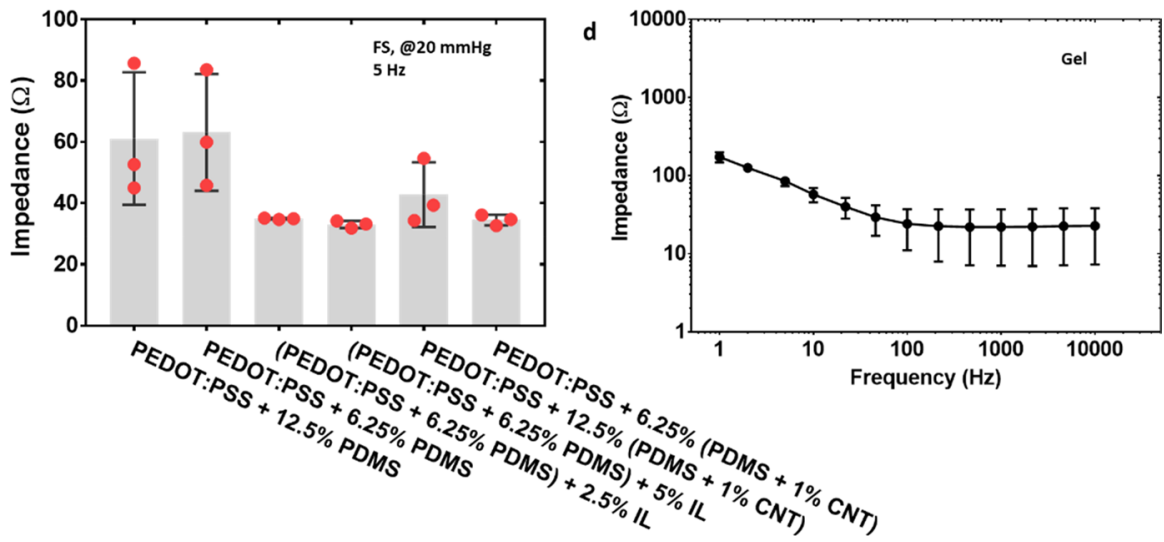

Fig. 5 The agar-electrode impedance of textile electrodes. a FS structure with various conductive coating materials in the frequency range of $1-10,000 \mathrm{~Hz}$. b FC structure with various conductive coating materials in the frequency range of $1-10,000 \mathrm{~Hz}$. c FS structure with PEDOT:PSS-contained coating in the frequency of $5 \mathrm{~Hz}$. The pressure was $20 \mathrm{mmHg}(\sim 2.66 \mathrm{kPa})$. d Wet gel electrode in the frequency range of 1-10,000 Hz 
enhanced ionic-to-electric coupling effect, leading to a better impedance match with human skin [29]. In addition, we found that the impedance of dry textile electrodes with PEDOT:PSS-contained coatings can be further reduced through the adding of CNT or ionic liquid (Fig. 5c). The CNT-induced enhancement can be because CNTs suppress the phase separation of PEDOT:PSS. Namely, the nanotubes establish electrical interconnections between the separate PEDOT:PSS (conductive phase) islands being dispersed in the insulating PSS-phase, thereby enhancing the electrical conductivity [37, 38]. On the other hand, ILs as a secondary dopant can induce the formation of a nanocrystallized fibrillar structure of PEDOT chains with extended planar confirmations and reduced $\pi-\pi$ interchain distances $[39,40]$. There is no impedance difference among PEDOT:PSS-contained coating with various concentrations of PDMS containing CNTs or IL (Fig. 5c).

\section{Effect of different conductive substrates (silver or carbon) and knitted structures (flat or raised}

\section{D)}

In this study, we selected the dry textile electrodes with carbon-contained coatings for investigation because PEDOT:PSS + PDMS-contained coatings cracked on all dry textile substrates (RS, FC, and RC) except the FS structure. These coatings can only be printed on FS structure. In contrast, carbon-contained coatings could be printed on both flat and raised 3D structures without cracks formation due to the strength of covalent bonds between carbon atoms. All tests were conducted at $5 \mathrm{~Hz}$ because the ECG spectrum has been reported to present optimal signal amplitudes at the frequency of $4-5 \mathrm{~Hz}$ [41]. All carbon-contained coatings showed a similar impedance level on those four types of electrode structures; only the FS structure with (carbon +5\%IL) coating showed relatively high value of impedance (Fig. 6a) but still within the acceptable impedance range (e.g., $\leq 10 \mathrm{k} \Omega$ ) of dry electrodes [7].

Dry textile electrodes have also shown a huge potential for epidermal electroencephalography (EEG) and electromyography (EMG) recording, in which tests were clinically conducted at higher sampling frequency ranges (e.g., $1 \mathrm{kHz}$ ) [36]. Therefore, we investigated the impedances of dry textile electrodes at the frequency of $1 \mathrm{kHz}$ as well. As

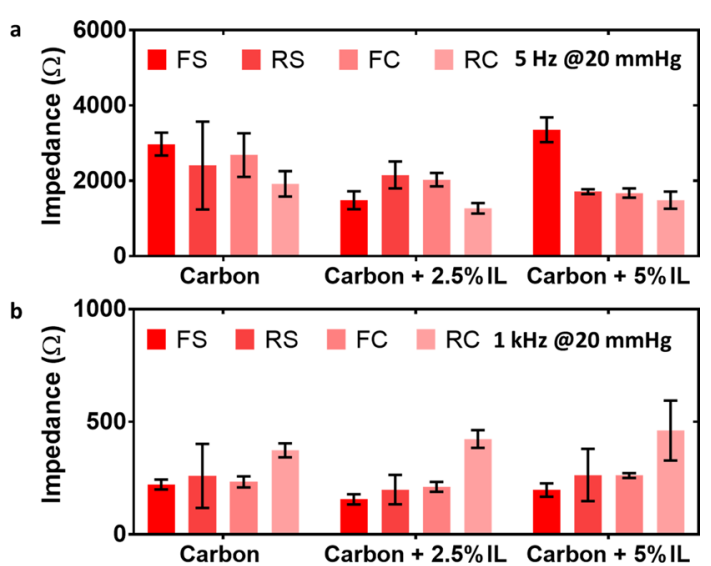

Fig. 6 The agar-electrode impedance of dry textile electrodes. Different conductive substrates (silver and carbon) and knitted structures (flat and raised 3D) at low frequency (a $5 \mathrm{~Hz})$ and high frequency $(\mathbf{b} 1 \mathrm{kHz})$ 
shown in Fig. 6b, all carbon-contained coatings showed similar impedance levels on FS, RS, and FC structures. Only carbon-contained coatings on the RC structure showed a relatively higher impedance (Fig. 6b).

\section{Effect of pressure}

Dry electrodes have shown great potentials for getting high-quality epidermal biopotential signals, including ECG, EMG, and EEG, in various conditions such as dry and wet skin, and during body movement [29]. Since dry textile electrodes do not have an adhesive, a proper pressure must be applied to maintain the skin-electrode contact to improve the quality of recorded biopotential signals [42]. Therefore, we investigated the effect of pressure on skin-electrode impedance. We selected flat textile substrates made of silver yarn since all the conductive coating materials listed in Table 1 could be successfully printed on this type of substrate without cracks. Pressures of $10 \mathrm{mmHg}, 20 \mathrm{mmHg}$, and $30 \mathrm{mmHg}(\sim 1.33 \mathrm{kPa}, \sim 2.66 \mathrm{kPa}$, and $3.99 \mathrm{kPa}$, respectively) were chosen, as they are within the optimal pressure range for compression applications of electrodes [34, 35]. We found that the pressure had little effect on the impedance of dry textile electrodes with different conductive coating materials (Fig. 7). Thereafter, the pressure of $20 \mathrm{mmHg}(\sim 2.66 \mathrm{kPa})$ was chosen for the following ECG measurement. This pressure has been shown to deliver the best signal fidelity for ECG applications [42].

\section{Effect of temperature}

In previous studies with gel electrodes, it has been shown that the resistive and capacitive components of the skin-electrode interface increase with decreasing temperature [43]. This observation was made for the temperature ranges $26-36{ }^{\circ} \mathrm{C}$. This change in impedance has been suggested to be correlated to the change in temperature, rather than changes in blood flow [43]. In this study, the temperature was kept at consistent room temperature of $23^{\circ} \mathrm{C}$, and no fluctuations were presented either to the agar sample

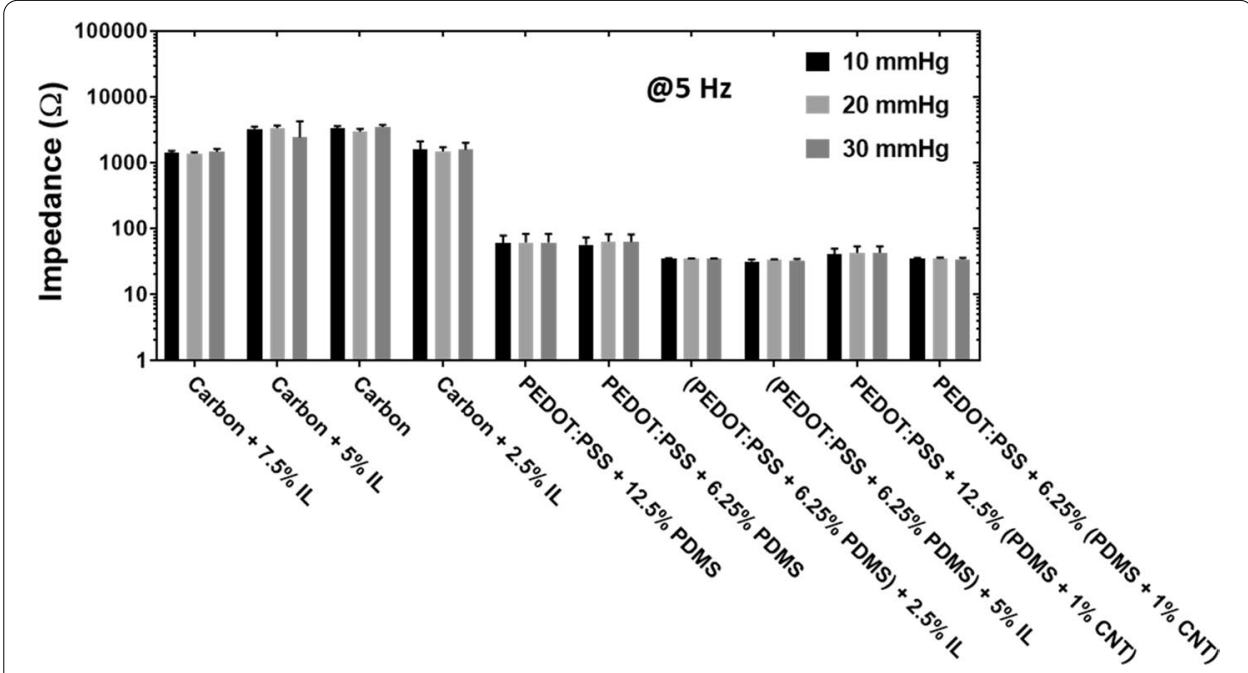

Fig. 7 Agar-electrode impedance of dry textile electrodes. FS structure at the pressures of $10 \mathrm{mmHg}$, $20 \mathrm{mmHg}$, and $30 \mathrm{mmHg}(\sim 1.33 \mathrm{kPa}, \sim 2.66 \mathrm{kPa}$, and $3.99 \mathrm{kPa}$, respectively). The frequency was at $5 \mathrm{~Hz}$ 
or the user being tested. Therefore, we did not account for or investigate the effect of temperature, considering the observed inverse linear relationship of skin-electrode impedance to changes in temperature [43].

\section{Long-term impedance stability}

The impedance stability performance of the dry electrodes over time is another important concern for the real application of smart garments [36]. We evaluated the deterioration of the dry textile electrodes by quantifying their impedance change within one month. The dry textile electrodes with IL/CNTs-contained coatings maintained their impedance well (Fig. 8). In contrast, dry textile electrodes with pure carbon coatings or PEDOT:PSS + PDMS coatings showed obvious impedance increases.

\section{Demonstration of ECG quality}

We evaluated the ECG quality on human skin using the electrodes shown in Fig. 8. Here, we selected dry textile electrodes in FS structure with carbon + 7.5\% IL coating, FS structure with PEDOT:PSS $+12.5 \%$ (PDMS $+1 \% \mathrm{CNT}$ ) coating, and FC structure with carbon $+7.5 \%$ IL coating for ECG monitoring due to their relative good longterm stability (Fig. 8). The dry electrodes were stored dry at room temperature for one month. Five replicates of each electrode were used in order to confirm the presented results. The electrodes were placed on the subject's arm as shown in Fig. 9a, along with two commercial disposable $\mathrm{Ag} / \mathrm{AgCl}$ electrodes as the gold standard simultaneously recorded as the second channel, and a third $\mathrm{Ag} / \mathrm{AgCl}$ electrode as the driven ground electrode. The pressure was applied at $20 \mathrm{mmHg}(\sim 2.66 \mathrm{kPa})$ through a stretchable compression band and measured with a PicoPress ${ }^{\circledR}$ sensor. Although the dry textile electrode in FS structure with PEDOT:PSS + 12.5\% (PDMS + 1\% $\mathrm{CNT}$ ) coating showed good long-term stability and an extremely low impedance $(\sim 50 \Omega$, Fig. 8), it did not present the best ECG signal quality (Fig. 9d). In contrast, the dry textile electrode in FS structure with carbon $+7.5 \%$ IL coating showed a relatively high impedance $(\sim 1500 \Omega$, Fig. 8$)$, but it presented a similar ECG spectrum (Fig. 8c) as the standard gel electrode (Fig. 9b). Conversely, the dry textile electrode in FC structure with carbon $+7.5 \%$ IL coating showed a relatively high impedance $(\sim 1500 \Omega$, Fig. 8), but it presented an ECG spectrum (Fig. 8e) with poor signal quality. In addition, we selected dry textile electrodes in FC structure with carbon coating

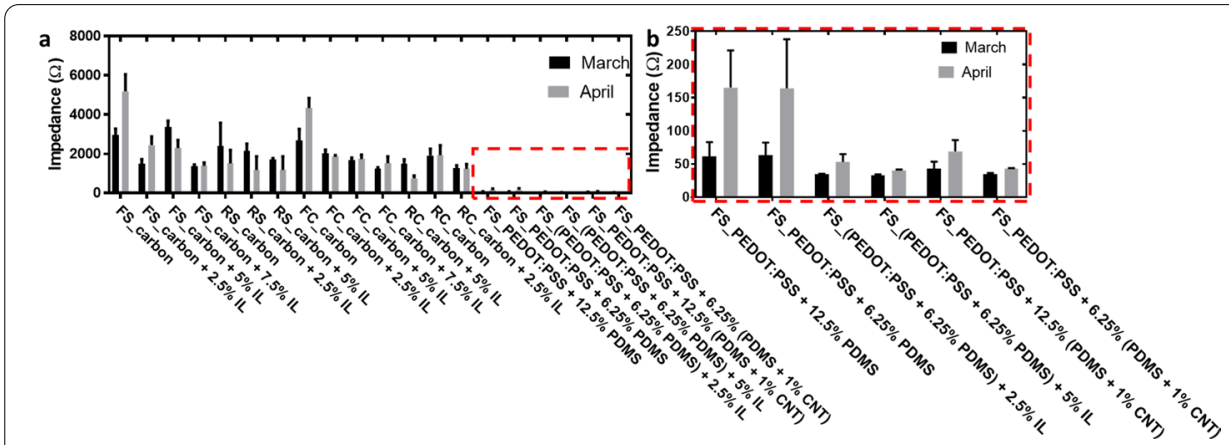

Fig. 8 Long-term impedance stability. a Dry textile electrodes with various coatings and substrates and $\mathbf{b}$ zoom-in of PEDOT:PSS-contained electrodes. The pressure was $20 \mathrm{mmHg}(\sim 2.66 \mathrm{kPa})$ 
a
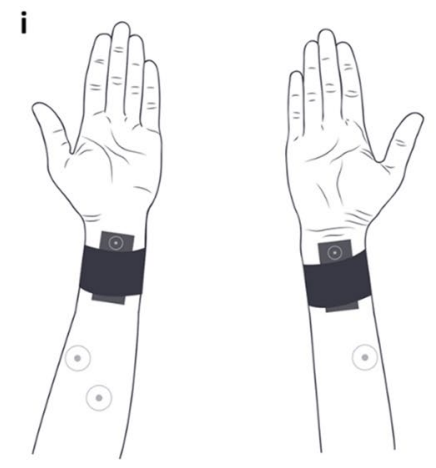

ii

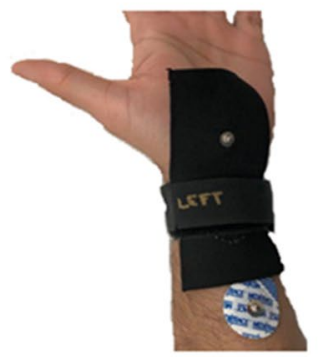

b
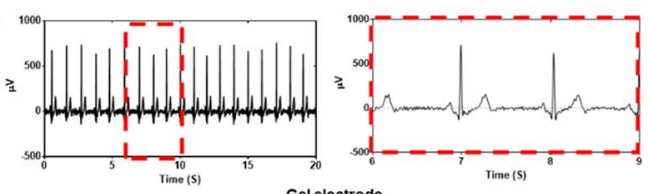

C
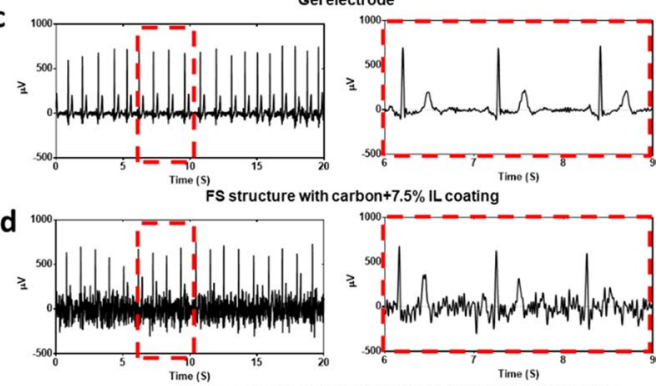

e

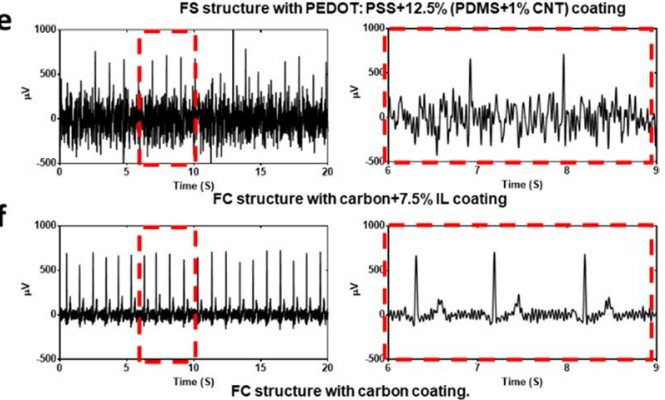

Fig. 9 ECG measurement. a ECG measurement setup (i) and photo of electrodes adhered to the skin (ii). Data of ECG monitoring using $\mathbf{b}$ gel electrode, $\mathbf{c}$ dry textile electrode in FS structure with carbon $+7.5 \% \mathrm{IL}$ coating. $\mathbf{d}$ Dry textile electrode in FS structure with PEDOT: PSS + 12.5\% (PDMS + 1\% CNT) coating. e Dry textile electrode in FC structure with carbon $+7.5 \% \mathrm{IL}$ coating. $\mathbf{f}$ Dry textile electrode in FC structure with carbon coating

as the representative electrodes without good long-term stability. We noticed that with a high impedance $(\sim 3000 \Omega$, Fig. 8$)$ and a poor long-term stability (with a $\sim 75 \%$ impedance increase in 30 days, Fig. 8), the dry textile electrode in FS structure with carbon coating showed a better ECG spectrum (Fig. 9f) than the FS structure with PEDOT:PSS + 12.5\% (PDMS + 1\% CNT) coating (Fig. 9b).

We further evaluated ECG signals based on the signal quality metrics of pSQI values, R-peak amplitudes, peak-to-peak amplitudes, and SNR values (Fig. 10). Although the impedance of dry textile electrodes varies based on the coating conductive materials, their PSQI values, R-peak amplitudes, and peak-to-peak amplitudes are all relatively stable and independent of the contact impedance. The relatively similar PSQI values indicate that the QRS complex was well-captured by all dry textile electrodes. We found that the dry textile electrode in FS structure with PEDOT:PSS + 12.5\% (PDMS + 1\% CNT) coating has a comparable impedance level to the standard gel electrodes but a significantly lower signal-to-noise ratio (SNR) value. In contrast, the dry textile electrode in FS structure with carbon $+7.5 \%$ IL coating showed a significantly higher impedance level than the standard gel electrodes but a comparable SNR value (Fig. 10). Therefore, in dry contact textile electrodes, lower impedance was not correlated to improvements in ECG signal performance, which is contrary to previous findings [7, 28, 29]. 


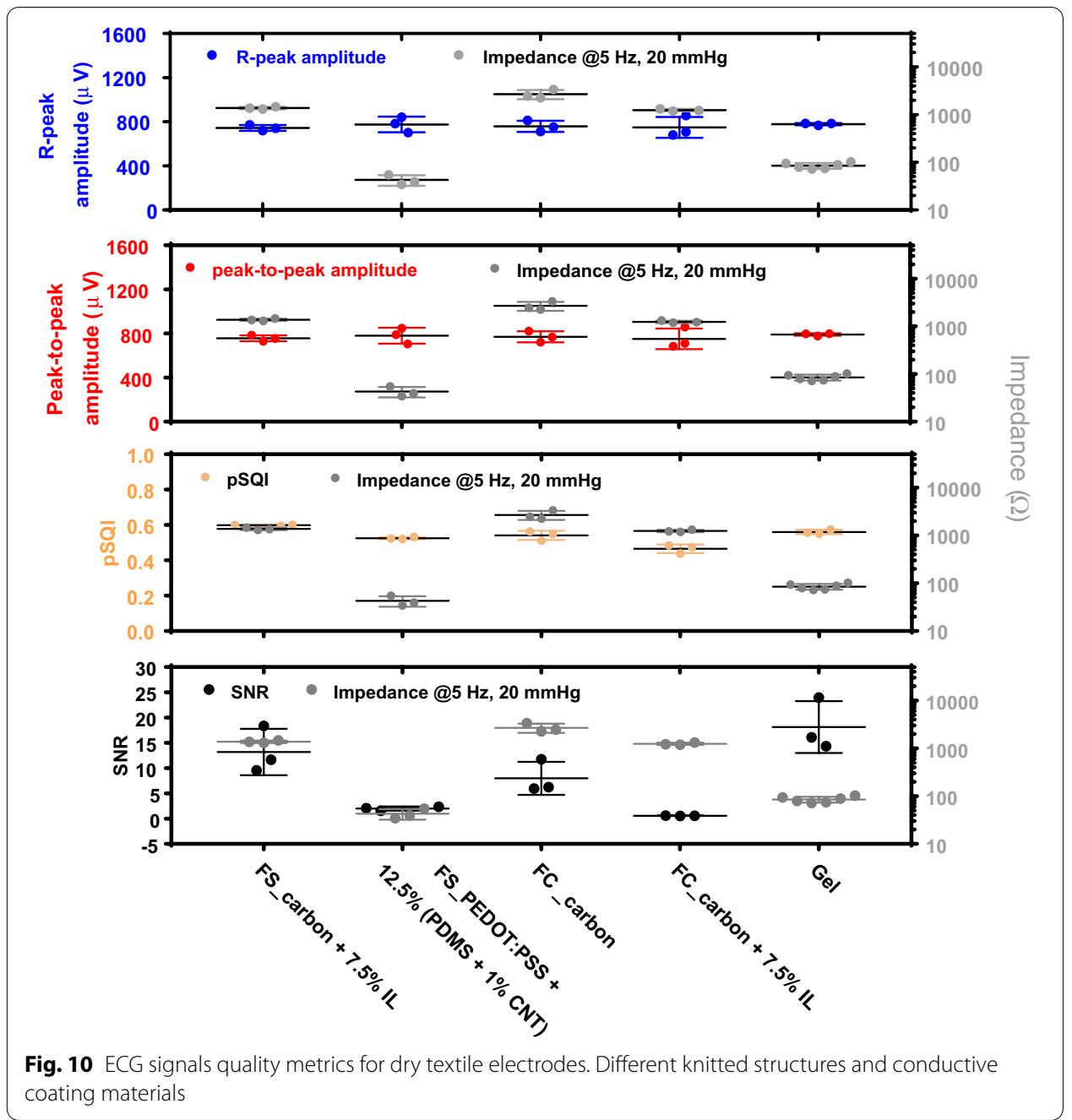

\section{Discussion}

In this work, we, for the first time, provided a standard testing procedure and framework for skin-electrode impedance measurement for the development of novel contact textile electrodes. From the experimental results, we found that carbon-contained conductive materials with proper concentration of IL (e.g., $\leq 5 \%$ ) showed good printability on all dry textile structures (i.e., FS, RS, FC and RC). The PEDOT:PSS + PDMS-contained conductive materials only showed printability on the FS structure. During impedance tests, we found the electrode impedance decreased as the increase of frequency; PEDOT:PSS-contained conductive coatings showed a gel-like electrode impedance, which is much smaller than that of carbon-contained conductive coatings. In addition, for carbon-contained conductive coatings, the electrode materials and structure have little impact on the electrode impedance. Similarly, pressure has no impact on the electrode impedance. As well we found dry textile electrodes with IL/CNTs-contained coatings showed good long-term impedance stability. During ECG measurements, the lower impedance of dry contact textile electrodes with PEDOT:PSS-contained conductive coatings was not correlated to improvements in ECG signal performance. 
Based on the results of this study, impedance cannot be used as a single factor to predict the performance of the materials or structures of textile-based dry contact electrodes for electrophysiological applications. The study highlights the importance of considering the electrophysiological application signal, in this case electrocardiography. To that effect, we have demonstrated the relation between electrode-skin impedance and signal quality was investigated using R-peak and peak-to-peak amplitudes as well as PSQI and SNR (Fig. 10). All these parameters are quantifiable attributes of an ECG signal which can provide insight into the performance of the textile-based dry contact electrode.

This work focused on studying the skin-electrode impedance of dry textile electrodes and its potential impact on ECG performance. Other performance of dry textile electrodes such as the motion artifact, electrodes' breathability and long-term skin irritation of current dry textile electrodes, are worth being explored. Their performance under sweating conditions and stretching while implemented in a textile form factor needs to be investigated for the practical usability considerations. Factors such as textile stretching and wash cycles would definitely impact the performance of dry electrodes. The results and finding of our research would allow us and other researchers to select the best performance dry-electrode formulation and textile substrate for their application and study their life-cycle performance using various wash and wear cycles. In our case, this would be investigated in our future studies depending on the application (ECG, EMG, EOG, and EEG monitoring) of dry electrodes and the textile form factor (sleeve, chest band, underwear, bra, tank top, head band, legging, etc.) since each of these form factors would require various amount of stretchability during wear cycles and different number of wash cycles it was not investigated in depth for this study. Also, it is worth exploring why higher concentrations of IL in the conductive material have a negative effect on the screen printing. In addition, potential biocompatibility and cytotoxicity considerations (mainly originated from the cation) of IL-contained dry textile electrodes need to be investigated over longterm wearing. Currently, there are many efforts pursuing the development of novel formulations of IL with large and bulky cations that can be entrapped in a polymeric matrix with stable and biocompatible features [8]. Before, during and immediately after application of dry electrodes, the skin irritation observation test was performed [44]. No skin irritation was observed for the individual who tested the electrodes for the duration of the testing. Though it is recommended that any materials selected for dry textile electrode long-term usage to be tested and certified based on ISO 10993-5, biological evaluation of medical devices. The current study measured the ECG performance using the dry textile electrode in FS structure with PEDOT:PSS $+12.5 \%$ (PDMS $+1 \% \mathrm{CNT}$ ) coating. More ECG tests need to be conducted on dry textile electrodes with gel-electrode-like low impedance to better understand how the low impedance determines the quality of ECG monitoring. In addition, our study focused on the ECG monitoring scenario, other biopotential monitoring (e.g., EMG, EEG, and EOG) using dry textile electrodes are worth being explored. These explorations are expected to provide more useful guidelines to design dry textile electrodes for other biopotential monitoring through a universal/standard evaluation protocol. 


\section{Conclusions}

In this paper, we studied and compared the impedance and performance of dry textile electrodes under different factors of substrate and structure, conductive coating materials, pressures, and frequencies. As well we explored their impedance durability for potential long-term applications in wearables. Unlike previous studies that focused on one conductive material across different testing platforms and parameters to evaluate the skin-electrode impedance, this is the first comprehensive study on a wide range of reported materials and their combinations using a skin model. The study has provided a standard testing procedure and framework for the classification of any newly developed materials, substrates or structures for dry contact electrodes, above and beyond dry contact textile electrodes. As well the study has provided a guideline for attributing the performance of dry textile electrodes in practical applications such as ECG monitoring. For example, a raised 3D electrode surface was expected to produce a lower impedance due to their better contact with the skin, while our experimental results did not indicate such a significant tendency. The pressure had little impact on the electrode impedance. In addition, from the impedance tests, we found the electrodes in FS structure with (PEDOT:PSS + 6.25\% PDMS) + 2.5\% IL coating, $($ PEDOT:PSS $+6.25 \%$ PDMS $)+5 \%$ IL coating, PEDOT:PSS $+12.5 \% \quad$ (PDMS $+1 \%$ CNT) coating, and PEDOT:PSS $+6.25 \%$ (PDMS $+1 \%$ CNT) coating showed a low impedance level $(\sim 50 \Omega)$, which is comparable the standard gel electrodes. However, the lower impedance of dry contact textile electrodes [e.g., in FS structure with PEDOT:PSS + 12.5\% (PDMS + 1\% CNT) coating] cannot guarantee a better ECG performance. In contrast, dry textile electrodes with high impedance (e.g., in FS structure with carbon $+7.5 \%$ IL coating, $\sim 1500 \Omega$ ), presented a high-quality ECG spectrum, similar to that of the standard gel electrode. Therefore, impedance alone should not be considered as the primary indicator for the quality of dry contact electrodes for biopotential recordings.

This study is a culmination of a number of efforts within the field of materials for smart textiles in continuous electrophysiological monitoring, specifically, electrocardiogram. This study is the first of its kind that contributes to the body of scientific knowledge by comparing the variety of materials found in the literature, serving as a comprehensive comparison of these proposed possibilities. These studies have been referenced and used as a logical framework for the selection of materials with the highest promise, towards a meaningful assessment of their efficacy and feasibility. The study not only assessed their electrical performance, but also provided an understanding of how these proposed materials can be produced for continuous monitoring applications, with overviews on their durability after being produced. As well, the study has explored the introduction of new variants through the inclusion of ionic liquids. This collective effort was designed as such to provide a thorough investigation in the field of dry contact textile electrodes, paving the way and encouraging further research in the field, for other electrophysiological applications such as EMG, EEG and EOG. Beyond new applications, there are continuing avenues for this paper, such as, biocompatibility/cytotoxicity considerations based on ISO standards, long-term material durability during wash, wear and tear that an everyday textile would undergo. Though these are beyond the scope of the project, and will be explored in future efforts. 


\section{Methods}

The aim of this study was the assessment of the electrical impedance property and performance of dry contact textile electrodes. This was done for a number of materials that have been highly reported and used for on body continuous monitoring applications, both in textiles and printed electronics. In this pursuit a number of the proposed materials that were tested did not successfully translate to textile substrates, which has been documented in the study. For the materials that were successfully implemented, impedance measurements were taken. The literature suggests, the difference between dry contact and conventional adhesive gel electrodes, lies within the skin-electrode impedance profile. In order to systematically approach this challenge, we created a standard testing procedure, which controlled parameters that could affect impedance, namely electrode size, contact pressure, and testing medium (agar). This standard testing procedure was also a helpful and useful outcome of this study, providing researchers a guideline for their future research, development and testing in this area.

\section{Fabrication of dry textile electrodes}

Using a flatbed knitting machine (Stoll, Reutlingen, Germany) silver-plated nylon and carbon-contained nylon conductive yarns (Myant Inc., Canada) were knitted into four different structures; flat textile electrodes made of silver yarn (FS Sample code), raised 3D textile electrodes made of silver yarn (RS Sample code), flat textile electrodes made of carbon yarn ( $F C$ Sample code) and raised 3D textile electrodes made of carbon yarn ( $R C$ Sample code).

These two conductive yarns (namely silver-plated and carbon-contained nylons) are the most common yarn-based conductive materials applicable in textile electrodes. They could also be mass-produced into the yarn form. Anisotropic conductive thermoplastic adhesive (EXP 2650-50, Creative Materials), carbon paste (DuPont ${ }^{\mathrm{TM}}$ PE671), carbon black (Cabot Corporation), carbon nanotube (CNT, SilQuan-C, NanoQuan), ionic liquid [1-Ethyl-3-methylimidazolium bis(trifluoromethylsulfonyl) imide, 99\%, IL-0023, Iolitec], PDMS (SYLGARD ${ }^{\mathrm{TM}}$ 184, Dow Corning Corporation), (Poly(3,4ethylenedioxythiophene)-poly(styrenesulfonate) (PEDOT:PSS, Orgacon EL-P501, Orgacon), Graphene (N006-P, Angstron Materials) and Neoprene pellets [Neoprene Polychloroprene, DuPont (pellets)] were used as ingredients of the different conductive pastes made for this study. The conductive pastes were either solely carbon-based or contained the polymers PEDOT:PSS, PDMS, and/or ionic liquid, and their detailed compositions are summarized in Table 1 and Additional file 1: Table S2. To make a homogeneous conductive paste, first the ingredients were mixed at $3500 \mathrm{rpm}$ for $30 \mathrm{~s}$ using a speed mixer [SpeedMixerTM (DAC 150.1 FV)]. The paste was placed on a custom-made silk screen mesh (156 mesh count with a template being $2.54 \mathrm{~cm}$ by $2.54 \mathrm{~cm}$ square, large enough to completely cover the circular textile electrode surface) and spread through the screen via a squeegee to form a smooth surface on a glossy-finish heat-transfer paper (Additional file 1: Figure S1). The curing condition of each layer depended on the composition of the conductive dry-electrode paste. The number of printing layers and curing conditions for each layer were per the recommendations of the materials supplier. Material compositions, number of ink layers used, and heat curing settings used in this study 
are given in Table 1 and Additional file 1: Table S2 with and without successful screen printing, respectively. The selection criteria of successful printing are based on the fact that printed electrodes maintain fidelity and mechanical durability when bent $180^{\circ}$ for 50 times. The optimal method for the screen printing of the conductive pastes was on a heat-transfer paper, rather than direct screen printing on a textile substrate. To allow for proper conductive adhesion to the textile substrate, an anisotropic conductive thermoplastic adhesive was printed on top of the cured conductive layers for paste formulations not containing PDMS. After printing and curing the required number of layers for each coating paste on a heat-transfer paper, the prints were heat-transferred $\left(250^{\circ} \mathrm{F}\right.$, for $30 \mathrm{~s}$, at $90 \mathrm{psi}$ ) from the paper to the textile, such that the adhesive was in contact with the conductive textile portion. Since PDMS has poor adhesive properties, pastes containing PDMS were printed on top of the cured layer of a thermoplastic adhesive and then manually peeled from the paper and applied to the textile substrate. The printed electrodes were then laminated onto the textile substrates, with the thermoplastic adhesive side of the electrode coming in contact with the textile substrate, and then adhered via heat-transfer. To complete the electrodes, a non-conductive adhesive film (BEMIS Company, Inc.) was applied as the last step via heat-transfer. This was designed as such to ensure an equal functional (conductive component) contact area for all electrodes, equivalent to a circle with a diameter of $2 \mathrm{~cm}$. As well, for all heat-transfers, the settings of temperature, time and pressure were kept the same to ensure consistency in process. This helped prevent non-homogenous factors that could impact the material characteristics, ultimately affecting performance and durability of the electrodes. This Fig. 2, h shows flat and raised 3D textile electrodes with screen printing of the conductive paste. All developed electrodes were then placed in a standard atmosphere for $24 \mathrm{~h}$ [relative humidity $(\mathrm{RH})=65 \pm 2 \%$ and $T=20 \pm 2{ }^{\circ} \mathrm{C}$ ] before their electrical properties were measured. Digital microscope (Oitez USB microscope) was used to evaluate the morphological characteristics of textile electrodes.

\section{Skin-electrode impedance measurement}

The skin-electrode impedance of the dry textile electrodes produced with various coatings was recorded using an Ivium Potentiostat (Ivium Technologies, Netherlands) in the frequency ranges from $1 \mathrm{~Hz}$ to $10 \mathrm{kHz}$. For consistency in the testing of electrodes, which can be significantly affected by intra- and inter-subject skin impedance variations, a human skin model optimized to match test results on human skin was created [33]. Briefly, this was achieved by mixing a solution of $4.5 \%$ agar, $0.97 \% \mathrm{NaCl}$, and deionized water on a hot plate until boiling, after which it was poured into a glass container and cooled until the mixture solidified. A new batch of agar was prepared using the mentioned recipe for each daily experimental and each agar batch was used for no longer than $7 \mathrm{~h}$. This was done to prevent drying of the agar simulated skin model, which would impact impedance recordings. Three dry textile electrodes were placed $7 \mathrm{~cm}$ apart on the agar skin model using the configuration reported in studies assessing skin-electrode contact impedance [12] (Additional file 1: Figure S2). With the 2-electrode test (Additional file 1: Figure S2b), the Counter (C) and Reference (R) cables are connected to Electrode 1. The Sense (S) and Working (W) cables are connected to Electrode 2. For the 3-electrode test (Additional file 1: Figure S2a), the configuration is the same except 
the Working cable is connected to Electrode 3. For both, the Ivium supplies constant amplitude current through the $\mathrm{W}$ and $\mathrm{C}$ cables, while simultaneously reading voltage through the $\mathrm{S}$ and $\mathrm{R}$ cables. The 2-electrode test obtains the impedance of the skin tissue (including subcutaneous tissue), which in this case would be agar, as well as Electrode 1 and Electrode 2. The 3-electrode test obtains the impedance of the skin tissue (including subcutaneous tissue), which in this case would be agar, and Electrode 1. Therefore, the impedance of Electrode 2 alone can be calculated by subtracting the impedance obtained by the 3-electrode test from the 2-electrode test.

The electrodes were given 5 min to settle on the agar before recording impedance characteristics. The electrode was considered settled when the impedance values ceased to continually increase or decrease with each measurement. A pressure (e.g., $20 \mathrm{mmHg}$ or $\sim 2.66 \mathrm{kPa}$ ) was applied on the electrodes, using a weight with the area of the weight in contact with the electrodes equal to that of the electrode face. This pressure is typically used in textile biopotential measurement to provide a secure and comfortable fit with good signal quality [42]. The electrode impedance of each category was measured at least on 3 samples, along with that of hydrogel electrodes measured as the control group.

\section{Biopotential electrocardiogram (ECG) measurements}

The electrodes were compared with gold standard hydrogel electrodes (Kendall, Covidien) for ECG measurement. ECG of $40 \mathrm{~s}$ duration was collected for each electrode to demonstrate the effect of coating on electrode performance for signal fidelity and illustrate the performance of the textile electrodes in comparison to hydrogel electrodes. ECG measurements were performed on one individual to minimize inter-subject impedance variability due to differences in skin impedance.

A low-power, 24-bit analog front-end ECG acquisition board (ADS1293, Texas Instruments) was used to collect ECG signals at $853 \mathrm{~Hz}$. The acquisition board has an instrumentation amplifier ( $3.5 \times$ gain), followed by a sigma-delta modulator, and digital filter. The chip contains three programmable fifth-order Sinc filters with decimation rates of 4 , 5 , and 6, respectively. All signals were filtered with two fourth-order Butterworth filters, first using a high-pass filter $\left(f_{\mathrm{c}}=0.5 \mathrm{~Hz}\right)$, then using a low-pass filter $\left(f_{\mathrm{c}}=50 \mathrm{~Hz}\right)$. The Pan-Tompkins algorithm was used to find R-peaks [45]. MATLAB R2016b was used for all post-processing. The following metrics for signal quality were extracted from the filtered ECG:

1. Amplitude: the distance, in $\mu \mathrm{V}$, from the baseline signal to each $\mathrm{R}$-peak. The baseline refers to the mean value of the entire signal.

2. Peak-to-peak amplitude: the distance, in $\mu \mathrm{V}$, between consecutive from the $\mathrm{S}$ - to the R-peak [46].

3. pSQI: the relative power of the QRS complex, $\frac{\int_{5 \mathrm{~Hz}}^{15} \mathrm{~Hz}(f) \mathrm{df}}{\int_{5 \mathrm{~Hz}}^{40 \mathrm{~Hz}} P(f) \mathrm{df}}[47]$.

4. BasSQI: the relative power of the baseline signal, $1-\frac{\int_{0 \mathrm{~Hz}}^{1} \mathrm{~Hz} P(f) \mathrm{df}}{\int_{0}^{40 \mathrm{~Hz}} P(f) \mathrm{df}}[47]$.

To ensure that the use of textile electrodes did not alter the shape of the frequency distribution of the measured ECG, Welch's estimated power spectral densities (PSDs) were compared using Pearson's correlation coefficient $R^{2}$ for each of the ECG samples. This 
would help mitigate the influence of confounding factors that can affect the signal quality or skin impedance between measurements, such as abrasion of the stratum corneum during removal and replacement of electrodes, motion, skin hydration, and sweating [48-52].

\begin{abstract}
Abbreviations
Ag/AgCl: Silver/silver chloride; ECG: Electrocardiography; CVD: Cardiovascular disease; ICP: Intrinsically conductive polymers; PEDOTPSS: Poly(3,4-ethylenedioxythiophene)-poly(styrenesulfonate); IL: Ionic liquid; PDMS: Polydimethylsiloxane; CNT: Carbon nanotube; FS: Flat textile electrodes made of silver yarn; RS: Raised 3D textile electrodes made of silver yarn; FC: Flat textile electrodes made of carbon yarn; RC: Raised 3D textile electrodes made of carbon yarn; EEG: Electroencephalography; EMG: Electromyography; SNR: Signal-to-noise ratio; RH: Relative humidity; NaCl: Sodium chloride; pSQI: The relative power of the QRS complex; BasSQI: The relative power of the baseline signal; PSD: Power spectral density.
\end{abstract}

\title{
Supplementary Information
}

The online version contains supplementary material available at https://doi.org/10.1186/s12938-021-00905-4.

Additional file 1: Figure S1. Electrode screen printing. (a) The screen-printing setup with the screen and template pattern, and red squeegee. (b) The resulting deposition of conductive paste on heat transfer paper. Figure S2. Ivium connection setup for impedance testing. (a) 3-electrode configuration (b) 2-electrode configuration. Figure S3. The agar-electrode impedance. (a) RS structure and (b) RC structure with various conductive coating materials in the frequency range of 1-10000 Hz. The pressure was $20 \mathrm{mmHg}(\sim 2.66 \mathrm{kPa})$. Table S1. Summary of current solutions to dry textile electrodes for biopotential monitoring. Table S2. Coating formulations unsuccessfully screen-printed onto dry textile electrodes.

\section{Authors' contributions}

MAM was the primary researcher devising the conception, acquisition, analysis, and composition. BY supported the analysis and composition. AS supported the conception and acquisition. EL supported the acquisition and analysis. LE, FA, and GS supported the acquisition. AM supported the conception, acquisition and analysis. BM supported the conception and analysis. MRP was the principal investigator. All authors read and approved the final manuscript.

\section{Funding}

Research funding from the Natural Sciences and Engineering Research Council of Canada NSERC Alliance-Mitacs Accelerate grant for paid internships. Research funding from the Natural Sciences and Engineering Research Council (NSERC) Postgraduate Scholarship-Doctoral (PGS D) for paid research.

\section{Availability of data and materials}

The datasets during and/or analysed during the current study are available from the corresponding author on reasonable request.

\section{Declarations}

Ethics approval and consent to participate

Institutional ethics approval was obtained and performed in accordance with the Declaration of Helsinki for the acquisition of data from human participants. For the acquisition and dissemination of human participant data, informed consent to participate in the study was obtained from participants.

\section{Competing interests}

The materials, equipment and machinery were provided in-kind by Myant Inc. Milad Alizadeh-Meghrazi, Ladan Eskandarian, Farhana Abbas, Gurjant Sidhu, Amin Mahnam were employees of Myant Inc. Binbin Ying, Alessandra Schlums, Emily Lam, Bastien Moineau were paid interns of Myant Inc. Research activities depicted in this publications adhere to the Good Publication Practice guidelines for pharmaceutical companies (GPP3).

\section{Author details}

${ }^{1}$ KITE Research Institute, Toronto Rehabilitation Institute - University Health Network (UHN), Toronto, ON, Canada. ${ }^{2}$ The Institute for Biomedical Engineering, University of Toronto, Toronto, ON, Canada. ${ }^{3}$ Department of Mechanical Engineering, McGill University, Montreal, QC, Canada. ${ }^{4}$ Department of Mechanical and Mechatronics Engineering, University of Waterloo, Waterloo, ON, Canada. ${ }^{5}$ Department of Materials Science and Engineering, University of Toronto, Toronto, ON, Canada. ${ }^{6}$ Department of Chemistry, University of Toronto, Toronto, ON, Canada. ${ }^{7}$ Department of Nanotechnology Engineering, University of Waterloo, Waterloo, ON, Canada. ${ }^{8}$ Myant Inc., Toronto, ON, Canada.

Received: 28 April 2021 Accepted: 2 July 2021

Published online: 12 July 2021 


\section{References}

1. Drew BJ, Califf RM, Funk M, Kaufman ES, Krucoff MW, Laks MM, et al. Practice standards for electrocardiographic monitoring in hospital settings: an American Heart Association scientific statement from the Councils on Cardiovascular Nursing, Clinical Cardiology, and Cardiovascular Disease in the Young: endorsed by the International Society of Computerized Electrocardiology and the American Association of Critical-Care Nurses. Circulation. 2004;110(17):2721-46.

2. NantesButton VLDS. Principles of measurement and transduction of biomedical variables. New York: Academic Press; 2015.

3. Chi YM, Jung T-P, Cauwenberghs G. Dry-contact and noncontact biopotential electrodes: methodological review. IEEE Rev Biomed Eng. 2010;3:106-19.

4. Ask P, ÖDerg P, Ödman S, Tenland T, Skogh M. ECG electrodes: a study of electrical and mechanical long-term properties. Acta Anaesthesiol Scand. 1979:23(2):189-206.

5. Tronstad C, Johnsen GK, Grimnes S, Martinsen $\varnothing \mathrm{G}$. A study on electrode gels for skin conductance measurements. Physiol Meas. 2010;31(10):1395.

6. Ying B, Wu Q, Li J, Liu X. An ambient-stable and stretchable ionic skin with multimodal sensation. Mater Horiz. 2020;7(2):477-88.

7. Zalar P, Saalmink M, Raiteri D, van den Brand J, Smits EC. Screen-printed dry electrodes: basic characterization and benchmarking. Adv Eng Mater. 2020;22(11):2000714.

8. Takamatsu S, Lonjaret T, Crisp D, Badier J-M, Malliaras GG, Ismailova E. Direct patterning of organic conductors on knitted textiles for long-term electrocardiography. Sci Rep. 2015;5(1):1-7.

9. Eskandarian L, Lam E, Rupnow C, Meghrazi MA, Naguib HE. Robust and multifunctional conductive yarns for biomedical textile computing. ACS Appl Electron Mater. 2020;2(6):1554-66.

10. Acar G, Ozturk O, Golparvar AJ, Elboshra TA, Böhringer K, Yapici MK. Wearable and flexible textile electrodes for biopotential signal monitoring: a review. Electronics. 2019;8(5):479.

11. Soroudi A, Hernández N, Wipenmyr J, Nierstrasz V. Surface modification of textile electrodes to improve electrocardiography signals in wearable smart garment. J Mater Sci Mater Electron. 2019;30(17):16666-75.

12. Castrillón R, Pérez JJ, Andrade-Caicedo H. Electrical performance of PEDOT: PSS-based textile electrodes for wearable ECG monitoring: a comparative study. Biomed Eng Online. 2018;17(1):1-23.

13. Catrysse M, Puers R, Hertleer C, Van Langenhove L, Van Egmond H, Matthys D. Towards the integration of textile sensors in a wireless monitoring suit. Sens Actuators A. 2004;114(2-3):302-11.

14. Ishijima M. Cardiopulmonary monitoring by textile electrodes without subject-awareness of being monitored. Med Biol Eng Comput. 1997;35(6):685-90.

15. Marquez JC, Seoane F, Välimäki E, Lindecrantz K. Comparison of dry-textile electrodes for electrical bioimpedance spectroscopy measurements. J Phys Conf Ser. 2010:224(1):012140.

16. Yoo J, Yan L, Lee S, Kim H, Yoo H-J. A wearable ECG acquisition system with compact planar-fashionable circuit boardbased shirt. IEEE Trans Inf Technol Biomed. 2009;13(6):897-902.

17. Sinha SK, Noh Y, Reljin N, Treich GM, Hajeb-Mohammadalipour S, Guo Y, et al. Screen-printed PEDOT: PSS electrodes on commercial finished textiles for electrocardiography. ACS Appl Mater Interfaces. 2017;9(43):37524-8.

18. Negru D, Buda C-T, Avram D. Electrical conductivity of woven fabrics coated with carbon black particles. Fibres Text East Eur. 2012;1(90):53-6.

19. Reyes BA, Posada-Quintero HF, Bales JR, Clement AL, Pins GD, Swiston A, et al. Novel electrodes for underwater ECG monitoring. IEEE Trans Biomed Eng. 2014;61(6):1863-76.

20. Yang K, Freeman C, Torah R, Beeby S, Tudor J. Screen printed fabric electrode array for wearable functional electrical stimulation. Sens Actuators A. 2014;213:108-15.

21. Chu M, Naguib HE. Soft flexible conductive CNT nanocomposites for ECG monitoring. Smart Mater Struct. 2021;30(6):065003

22. Zhang Y, Liang B, Jiang Q, Li Y, Feng Y, Zhang L, et al. Flexible and wearable sensor based on graphene nanocomposite hydrogels. Smart Mater Struct. 2020;29(7):075027.

23. Khan S, Lorenzelli L. Recent advances of conductive nanocomposites in printed and flexible electronics. Smart Mater Struct. 2017;26(8):083001.

24. Qin H, Li J, He B, Sun J, Li L, Qian L. Novel wearable electrodes based on conductive chitosan fabrics and their application in smart garments. Materials. 2018;11(3):370

25. Zhang H, Li W, Tao X, Xu P, Liu H, editors. Textile-structured human body surface biopotential signal acquisition electrode. In: 2011 4th international congress on image and signal processing. IEEE; 2011.

26. Pola T, Vanhala J, editors. Textile electrodes in ECG measurement. In: 2007 3rd international conference on intelligent sensors, sensor networks and information. IEEE; 2007.

27. Liu S, Ma K, Yang B, Li H, Tao X. Textile electronics for VR/AR applications. Adv Funct Mater. 2020. https://doi.org/10.1002/ adfm.202007254

28. Maji S, Burke MJ. Establishing the input impedance requirements of ECG recording amplifiers. IEEE Trans Instrum Meas. 2019;69(3):825-35

29. Zhang L, Kumar KS, He H, Cai CJ, He X, Gao H, et al. Fully organic compliant dry electrodes self-adhesive to skin for longterm motion-robust epidermal biopotential monitoring. Nat Commun. 2020;11(1):1-13.

30. Marozas V, Petrenas A, Daukantas S, Lukosevicius A. A comparison of conductive textile-based and silver/silver chloride gel electrodes in exercise electrocardiogram recordings. J Electrocardiol. 2011;44(2):189-94.

31. Yao S, Zhu Y. Nanomaterial-enabled dry electrodes for electrophysiological sensing: a review. JOM. 2016;68(4):1145-55.

32. Tseghai GB, Mengistie DA, Malengier B, Fante KA, Van Langenhove L. PEDOT: PSS-based conductive textiles and their applications. Sensors. 2020;20(7):1881.

33. Idrissi A, Kaiser M, Albrecht S, Richert S, Gries T, Blaeser A. Development of a test bench for the characterization of movement artifacts in smart textile systems. Int J Bioelectromagn. 2018:20(1):80-3.

34. MacRae BA, Cotter JD, Laing RM. Compression garments and exercise. Sports Med. 2011;41(10):815-43.

35. Lawrence D, Kakkar V. Graduated, static, external compression of the lower limb: a physiological assessment. Br J Surg. 1980:67(2):119-21. 
36. Leleux P, Johnson C, Strakosas X, Rivnay J, Hervé T, Owens RM, et al. Ionic liquid gel-assisted electrodes for long-term cutaneous recordings. Adv Healthc Mater. 2014;3(9):1377-80.

37. Mustonen T, Kordás K, Saukko S, Tóth G, Penttilä JS, Helistö P, et al. Inkjet printing of transparent and conductive patterns of single-walled carbon nanotubes and PEDOT-PSS composites. Phys Status Solidi (b). 2007;244(11):4336-40.

38. Park J, Lee A, Yim Y, Han E. Electrical and thermal properties of PEDOT: PSS films doped with carbon nanotubes. Synth Met. 2011;161(5-6):523-7.

39. Kee S, Kim N, Kim BS, Park S, Jang YH, Lee SH, et al. Controlling molecular ordering in aqueous conducting polymers using ionic liquids. Adv Mater. 2016;28(39):8625-31.

40. Teo MY, Kim N, Kee S, Kim BS, Kim G, Hong S, et al. Highly stretchable and highly conductive PEDOT: PSS/ionic liquid composite transparent electrodes for solution-processed stretchable electronics. ACS Appl Mater Interfaces. 2017;9(1):819-26.

41. Razzaq N, Butt M, Salman M, Ali R, Sadiq I, Munawar K, et al., editors. Self tuned SSRLS filter for online tracking and removal of power line interference from electrocardiogram. In: 2013 5th international conference on modelling, identification and control (ICMIC). IEEE; 2013.

42. Cömert $\mathrm{A}$, Honkala $\mathrm{M}$, Hyttinen J. Effect of pressure and padding on motion artifact of textile electrodes. Biomed Eng Online. 2013;12(1):1-18.

43. Smith DC. Effects of skin blood flow and temperature on skin-electrode impedance and offset potential: measurements at low alternating current density. J Med Eng Technol. 1992;16(3):112-6.

44. Bareket L, Inzelberg L, Rand D, David-Pur M, Rabinovich D, Brandes B, et al. Temporary-tattoo for long-term high fidelity biopotential recordings. Sci Rep. 2016:6(1):25727.

45. Pan J, Tompkins WJ. A real-time QRS detection algorithm. IEEE Trans Biomed Eng. 1985;3:230-6.

46. Norland K, Sveinbjornsson G, Thorolfsdottir RB, Davidsson OB, Tragante V, Rajamani $\mathrm{S}$, et al. Sequence variants with large effects on cardiac electrophysiology and disease. Nat Commun. 2019;10(1):1-10.

47. Clifford G, Behar J, Li Q, Rezek I. Signal quality indices and data fusion for determining clinical acceptability of electrocardiograms. Physiol Meas. 2012;33(9):1419.

48. Beckmann L, Neuhaus C, Medrano G, Jungbecker N, Walter M, Gries T, et al. Characterization of textile electrodes and conductors using standardized measurement setups. Physiol Meas. 2010;31(2):233.

49. Besio W, Prasad A, editors. Analysis of skin-electrode impedance using concentric ring electrode. In: 2006 international conference of the IEEE engineering in medicine and biology society. IEEE; 2006.

50. Medrano G, Ubl A, Zimmermann N, Gries T, Leonhardt S, editors. Skin electrode impedance of textile electrodes for bioimpedance spectroscopy. In: 13th international conference on electrical bioimpedance and the 8 th conference on electrical impedance tomography. Springer; 2007.

51. Li G, Wang S, Duan YY. Towards gel-free electrodes: a systematic study of electrode-skin impedance. Sens Actuators B Chem. 2017;241:1244-55.

52. Sunaga T, Ikehira H, Furukawa S, Shinkai H, Kobayashi H, Matsumoto Y, et al. Measurement of the electrical properties of human skin and the variation among subjects with certain skin conditions. Phys Med Biol. 2001;47(1):N1 1.

53. Paul G, Torah R, Beeby S, Tudor J. The development of screen printed conductive networks on textiles for biopotential monitoring applications. Sens Actuators A. 2014;206:35-41.

54. Myers AC, Huang H, Zhu Y. Wearable silver nanowire dry electrodes for electrophysiological sensing. RSC Adv. 2015:5(15):11627-32

55. Xu F, Zhu Y. Highly conductive and stretchable silver nanowire conductors. Adv Mater. 2012;24(37):5117-22.

56. Lam CL, Saleh SM, Yudin MBM, Harun FK, Sriprachuabwong C, Tuantranont A, et al., editors. Graphene ink-coated cotton fabric-based flexible electrode for electrocardiography. In: 20175 th international conference on instrumentation, communications, information technology, and biomedical engineering (ICICI-BME). IEEF; 2017.

57. Celik N, Manivannan N, Strudwick A, Balachandran W. Graphene-enabled electrodes for electrocardiogram monitoring. Nanomaterials. 2016;6(9):156.

58. Yapici MK, AlkhidirT, Samad YA, Liao K. Graphene-clad textile electrodes for electrocardiogram monitoring. Sens Actuators B Chem. 2015;221:1469-74.

59. Hallfors N, Alhawari M, Abi Jaoude M, Kifle Y, Saleh H, Liao K, et al. Graphene oxide: nylon ECG sensors for wearable loT healthcare—nanomaterial and SoC interface. Analog Integr Circ Sig Process. 2018;96(2):253-60.

60. Chen Y-H, De Beeck MO, Vanderheyden L, Carrette E, Mihajlović V, Vanstreels K, et al. Soft, comfortable polymer dry electrodes for high quality ECG and EEG recording. Sensors. 2014;14(12):23758-80.

61. Peng H-L, Liu J-Q, Tian H-C, Xu B, Dong Y-Z, Yang B, et al. Flexible dry electrode based on carbon nanotube/polymer hybrid micropillars for biopotential recording. Sens Actuators A. 2015;235:48-56.

62. Liu B, Chen Y, Luo Z, Zhang W, Tu Q, Jin X. A novel method of fabricating carbon nanotubes-polydimethylsiloxane composite electrodes for electrocardiography. J Biomater Sci Polym Ed. 2015;26(16):1229-35.

63. Lam CL, Rajdi NNZM, Wicaksono DH, editors. MWCNT/cotton-based flexible electrode for electrocardiography. In: Sensors, 2013 IEEE. IEEE; 2013.

64. Lidón-Roger JV, Prats-Boluda G, Ye-Lin Y, Garcia-Casado J, Garcia-Breijo E. Textile concentric ring electrodes for ECG recording based on screen-printing technology. Sensors. 2018;18(1):300.

65. Pani D, Dessì A, Saenz-Cogollo JF, Barabino G, Fraboni B, Bonfiglio A. Fully textile, PEDOT: PSS based electrodes for wearable ECG monitoring systems. IEEE Trans Biomed Eng. 2015;63(3):540-9.

66. Bihar E, Roberts T, Saadaoui M, Hervé T, De Graaf JB, Malliaras GG. Inkjet-printed PEDOT: PSS electrodes on paper for electrocardiography. Adv Healthc Mater. 2017;6(6):1601167.

\section{Publisher's Note}

Springer Nature remains neutral with regard to jurisdictional claims in published maps and institutional affiliations. 